\title{
SUBDURAL HAEMATOMA IN A PATIENT WITH MENINGIOMA
}

\author{
Paulo Valdeci Worm 7 , Marcelo Paglioli Ferreira ${ }^{2}$, Nelson Pires Ferreira ${ }^{3}$, Fernanda Cechetti ${ }^{4}$
}

Non-traumatic subdural haematomas are very rare especially those associated with intracranial meningiomas'. Intracranial tumors hemorrhage occurs in $39 \%$ of all cases. They are a malignant pathology such as metastatic tumors or gliomas. They usually present with seizures hemiparesis visual field loss aphasia or other focal sumptoms.

We report a case of meningioma of the skull base associated with subdural haematoma after a Valsava maneuver.

\section{CASE}

A 64-year old male patient, smoker, had a loss of consciousness with mild headache during a spirometric exam. He was medicated with analgesics and remained with headache for 12 hours and was discharged from hospital. He persisted with chronic headache and developed a gradual confusional state and left

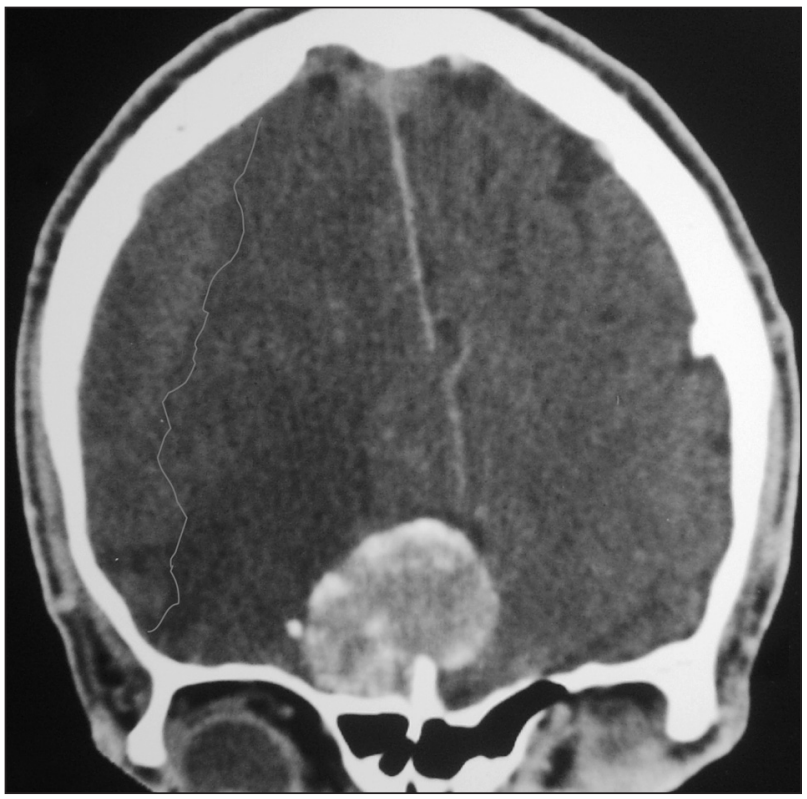

Fig 1. CT of skull showing meningioma of falx with adjacent edema and subdural collection in homolateral side. CT shows the brain shift and edema. hemiparesia. After the initial episode, five days after the spirometric test, he returned to the hospital for a computed tomography (CT) of the head (Figs 1 and 2). The CT showed a right subdural haematoma with a fresh clot in the anterior skull base with bone thickness of the anterior fossa besides meningioma of the falx with adjacent edema.

The patient had no prior history of trauma or alcoholism. He was using (AAS) $200 \mathrm{mg} /$ day. Coagulation tests were normal.

A right frontal osteoplastic craniotomy was performed draining the haematoma, and during the same surgical procedure the tumor was removed using microscopy.

A dark, thick, red blood clot was drained. During the surgical drainage of the subdural haematoma two findings were observed by the surgeon: 1 ) the homolateral side of the subdural haematoma presented with greater vascular density. 2) the clot surface on this side showed a variable aspect, suggesting ac-

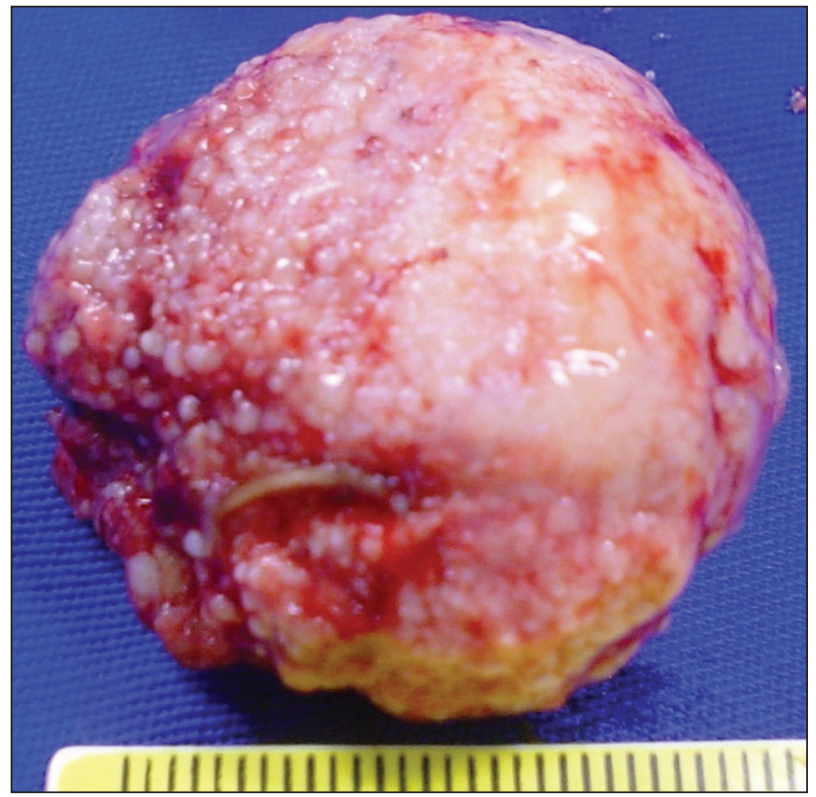

Fig 2. Tumor with two differents areas: plain and homogenic surface and another showing wrinkled aspect and higher vascular density with macroscopic homolateral haematoma.

\section{HEMATOMA SUBDURAL EM PACIENTE COM MENINGIOMA}

Hospital São José, Santa Casa de Misericórdia de Porto Alegre, Porto Alegre RS, Brazil: ${ }^{M D} ;{ }^{2} \mathrm{MD} ;{ }^{3} \mathrm{MD}$, PhD, Professor of Neurological Surgery, Department of Neurosurgery, Hospital São José - Santa Casa de Misericórdia de Porto Alegre, Porto Alegre RS, Brazil; ${ }^{4}$ MD, Programa de Pós Graduação em Ciências Biológicas-Neurociências, Universidade Federal do Rio Grande do Sul, RS, Brazil.

Received 17 September 2008, received in final form 19 December 2008. Accepted 13 March 2009.

Dr. Paulo Valdeci Worm - Rua Carlos Trein Filho 550 / 403 - 90450-120 Porto Alegre RS - Brasil. E-mail: pauloworm@hotmail.com 


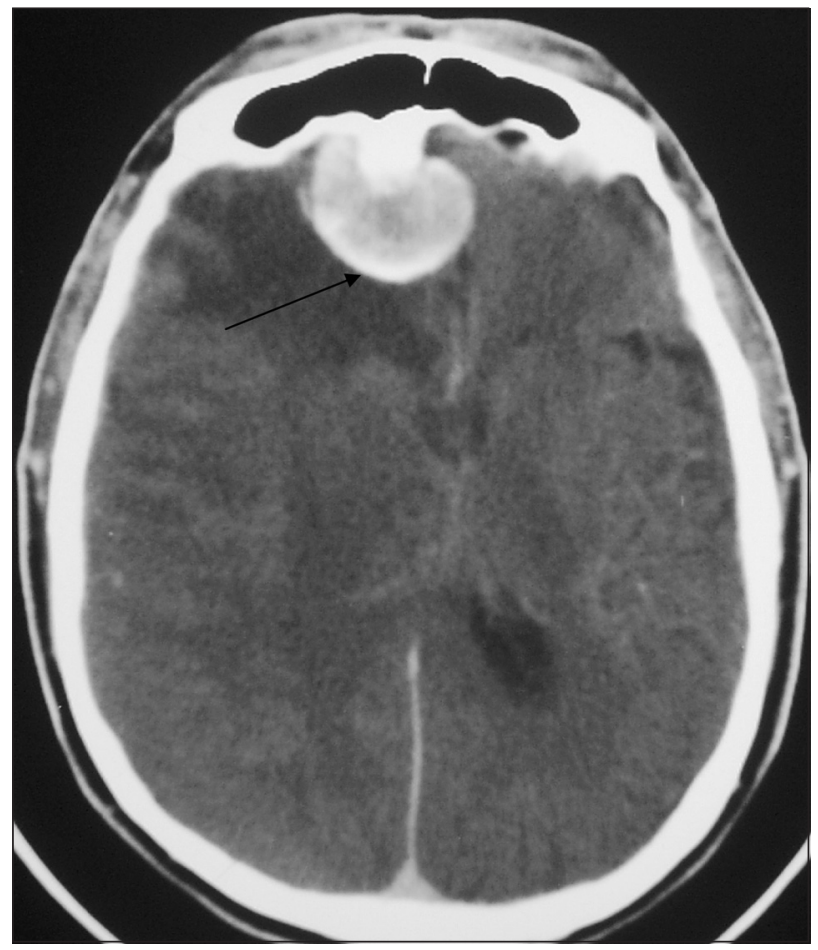

Fig 3. CT with contrast showing the meningioma and homolateral subdural haematoma area with higher capitation (black arrow).

tive bleeding (Fig 3). An en bloc surgical excision of the clot and the tumor were performed. The histopathological diagnosis was meningeoma. Right after drainage his motor state improved but he remained confused. He remained in hospital for seven days. When he was discharged from hospital he was partially oriented and had no focal neurological deficit.

\section{DISCUSSION}

Benign intracranial tumors like meningiomas rarely present with hemorrhage and the reported incidence is $1.3 \%$ of all meningiomas ${ }^{2}$. The higher incidence of this association was found by Niiro et al. ${ }^{2}$ who described six cases of hemorrhage in a series of 298 patients with intracranial meningiomas (2\%).

The physiopatological mechanisms of this association are not yet well understood, but several hypotheses have been proposed to explain the formation of a spontaneous hemorrhage in patients with intracranial meningioma: 1) distension of the feeding vessels and weakening of the thin walls with tumor growth and consecutive rupture; 2) stretching of subdural bridging veins caused by tumor expansion; 3) rupture of abnormal tumor vessels; 4) direct invasion of the tumor and disruption of blood vessels; 5) formation of neo-membranes, like chronic subdural haematoma caused by a dural reaction to the tumor; 6 ) vasoactive substances released by the meningioma.

Another hypothesis proposed by Gruskiewicz et al. is that rapid tumor growth and venous thrombosis leading to tumor necrosis have been implicated. Tumor necrosis can cause direct breakdown of the tumor vessels and subsequent hemorrhage; tortuous feeding arteries that are less resistant to blood pressure changes and susceptible to rupture are implicated ${ }^{3}$.

However, none of the proposed mechanisms alone can explain the phenomenon. The combination of several physiopathogenic factors should be seen as the most common event.

Other factors, such as tumor histological subtype, location, age and sex of the patient do not show significant relationship with the hemorrhagic event ${ }^{2}$. Subdural haematoma is not related to the presence of systemic arterial hypertension or tumor location. Some authors have emphasized a high risk of hemorrage in angioblastic and malignant meningiomas'.

It is conceivable that the localization of the tumor in the cerebral convexity increases the risk of hemorrhage. Chaskis et al. ${ }^{4}$ described 20 cases of hemorrhagic meningiomas. Eleven were situated in the convexity. Other authors have hypothesized that the development of a meningioma could be secondary to chronic irritation of the meninges exerted by chronic subdural haematoma. ${ }^{5}$.

Intracranial hemorrhage has less associations with benign tumors than with malignant ones ${ }^{5}$. Malignant metastatic melanoma, choriocarcinoma and bronchogenic carcinoma are associated with intracranial hemorrhage and are widely discussed in the literature. Chronic subdural haematoma is rarely associated with meningioma.

Itoyama et al. ${ }^{6}$ described a patient with bilateral subdural haematoma with a left sphenoid wing meningioma. Sinha and Dharker ${ }^{7}$ presented a case of a meningioma of the right occipital convexity associated with a left frontoparietal subdural haematoma. Pozzi et al. ${ }^{8}$ reported a right hemisphere convexity subdural haematoma associated left parietal meningioma. This contralateral injury is highly controversial. It could be due to small traumas caused by convulsions in clinical seizures of meningiomas.

Tanaka et al. ${ }^{9}$ correlated the localization and the histological subtype of meningiomas and a higher probability of the association with chronic subdural haematoma.

Some CT findings can help correlate a meningioma with subdural haematoma, such as: 1) bone alteration 2) focal cerebral distortion and cerebral perifocal edema due to the presence of meningioma inside the subdural clot collection 3) potential differences in the signal density betwen the different areas measured for the Hounsfield scale.

In the case reported we found bone alterations at the meningioma implantation site, evidence of a rich and vascular density in the homolateral side far from the haematoma; moreover, we found more macrocospic characteristic changes on this side. 
These findings call attention to the fact that the possible etiology of the hemorrhage had its genesis in a Valsalva maneuver that ruptured during the manipulation of forced expiration triggered for the spirometric examination. The patient probably already had the intracranial haematoma, and the use of AAS may have been one of the facilitating factors. This has not yet been described in the literature. The mechanisms of haematoma formation associated with the Valsalva maneuver are poorly understood but Shojima et al..$^{10}$ mentioned that events such as seizures or a rapid change in blood pressure may also act as etiologic agents for hemorrhage. In the CT the homolateral area near the haematoma was more clearly shown by the contrast beyond the presence of surrounding cerebral edema (Fig 3).

Although several hypotheses have been described to explain the bleeding in meningiomas, so far this event has been rare. We do not have a satisfactory explanation for this phenomenon. Besides observations of the histological properties and radiological change, possibly macroscopic observation of the tumor and the adjacent vasculature during surgery could, in future, help us reveal the histopathogenesis of the phenomenon.

\section{REFERENCES}

1. Helle TL, Conley FK. Haemorrhage associated with meningioma: a case report and review of the literature. J Neurol Neurosurg Psychiatry 1980;43:725-729.
2. Niiro M, Ishimaru $\mathrm{K}$, Hirano $\mathrm{H}$, et al. Clinico-pathological study of meningiomas with haemorrhagic onset. Acta Neurochir (Wien ) 2003;145:767-772.

3. Bloomgarden GM, Byrne TN, Spencer DD, et al. Meningioma associated with aneurysm and subarachnoid hemorrhage: case report and review of the literature. Neurosurgery 1987;20:24-26.

4. Chaskis C, Raftopoulos C, Noterman J, et al. Meningioma associated with subdural haematoma: report of two cases and review of the literature. Clin Neurol Neurosurg 1992;94:269-274.

5. Baskinis N, Grotenhuis A, Wandt H. Chronic subdural hematoma associated with an intracapsular meningioma: case report and short review of the literature. J Neurosurg Sci 1984;28:17-23.

6. Itoyama $\mathrm{Y}$, Fukumura A, Itoh $\mathrm{Y}$, et al. [Primary brain tumor complicating subdural hematoma]. No To Shinkei 1987;39:1157-1161.

7. Sinha VD, Dharker SR. Meningioma associated with contralateral chronic subdural haematoma: a short report. Neurol India 2001;49:204-206.

8. Pozzi M, Dario A, Marra A, et al. Associated chronic subdural haematoma and meningeal neoplasm: two case reports. J Neurosurg Sci 1993;37:113-117.

9. Tanaka N, Yamamoto M, Jimbo M, et al. Meningioma associated with chronic subdural hematoma and meningothelial cell cluster within the hematoma capsule: case report. Neurol Med Chir (Tokyo) 1994;34:176-179.

10. Shozima K, Hayashi T, Higashihama H. Cystic meningioma associated with intratumor and subarachnoid hemorrhage during embolization: a case report. No ShinkeiGeka 1986;14: 919-924. 